\title{
Optimal placement of phasor measurement units by genetic algorithm
}

\author{
Allagui B., Marouani I., Hadj Abdallah H. \\ ENIS, Dép. Génie Electrique \\ Email address: \\ allagui5555@yahoo.fr (A. B.), ismailmarouani@yahoo.fr (M. I.), hsan.haj@enis.rnu.tn (H. Abdallah H.)
}

\section{To cite this article:}

Allagui B., Marouani I., Hadj Abdallah H.. Optimal Placement of Phasor Measurement Units by Genetic Algorithm. International Journal of Energy and Power Engineering. Vol. 2, No. 1, 2013, pp. 12-17. doi: 10.11648/j.ijepe.20130201.12

\begin{abstract}
Monitoring and supervision of power systems are provided by the control center, whose role is the design, coordination and network management. This paper presents a control technique based on the implantation of measurement units at the network buses. This technique should meet two requirements: ensure the complete system observability and find the optimal locations of PMUs with the minimum cost. The problem was formulated as a mono-objective optimization problem and its resolution was made by implementing a genetic algorithm (GA). The proposed method is tested on three tests networks and the results are compared with other resolution techniques. The simulation results ensure the complete system observability and validate the presented technique.
\end{abstract}

Keywords: PMU, Optimal Placement, Complete System Observability, Genetic Algorithm

\section{Introduction}

The electrical power networks are continuously pushed to function at the limit of their stability. This is due to several economic, ecological, and technical constraints. In addition to these constraints, the opening of new markets increases the power consumption as well as the complexity of interconnections between the electrical power networks, thus, creating an important energy exchanges on the network gridlines. The exchanges on the network at the moment of an imbalance are visible by the oscillation of the power transiting on the network gridlines. This oscillation limits energy production of the machines and surpasses the generators' capacity. This fact highlights the importance of the monitoring of electrical power networks using adequate and modern methods.

Currently, many devices are developed to provide near instantaneous measurements (phase, current, and voltage). Such devices are called Phasor Measurement Units (PMU), [1-2]. This technology can provide a precise description of the real time state of the network operating conditions; thus, providing its total observability [3-4], correcting the errors related to mathematical representation, and improving the estimation of these conditions. The bus of the electrical power networks guarantees the visualization of the system which assists the operator to implement an automatic plan to maintain the stability of the network. That also reduces the number of monitoring personnel, as well as the risks of damage to the equipment in case of a sudden blackout.

The installation of PMUs in the electrical supply networks resulted in the development of several algorithms to determine the optimal placements of these devices. Such algorithms must be cost effective therefore using the minimum of these devices to ensure the total observability of the entire network.

The objective of this work consists in developing a MATLAB program that is based on the genetic algorithms to find the optimal placement of PMUs. The genetic algorithm method was tested on three network-tests, and the results were compared with other algorithms. The results of simulation prove that an optimal placement of PMUs ensures a total observability of the network and thus confirming the efficacy of genetic algorithm [5].

Reference [6] presents a detailed analysis of the required synchronization accuracy of several phasor measurement applications.

Figure 1 shows a hardware block diagram of a phasor measurement unit. The anti-aliasing filter is used to filter out from the input waveform frequencies above the $\mathrm{Ny}$ quist rate. The phase locked oscillator converts the GPS1 pulse per second into a sequence of high-speed timing pulses used in the waveform sampling. The microprocessor executes the DFT phasor calculations. Finally, the phasor is timestamped and uploaded to a collection device known as a data concentrator. An IEEE standard format now exists 
for real time phasor data transmission [7].

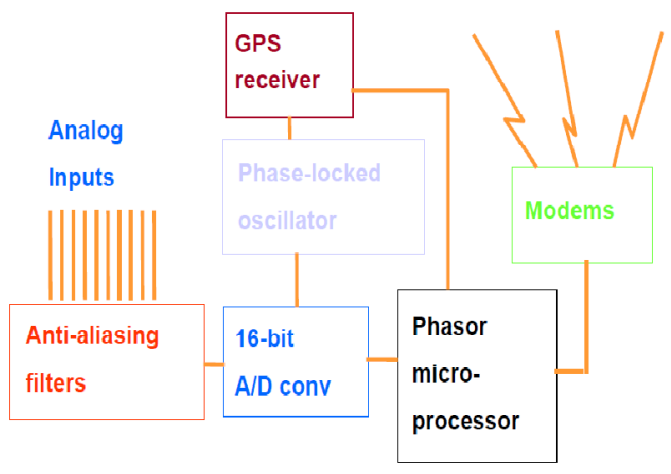

Figure 1. Phasor Measurement Unit Hardware Block Diagram

\section{Terms and Concepts of Observability}

The definition of certain terminologies is necessary to better understand Observability [8]:

A directly observable node is a node where a PMU is placed to measure the amplitude and phase of the voltage.

A node, without PMU, is calculable provided that there is a connection in at least one PMU.

A node is unobservable when one or more variables needed to determine its condition is missing.

The complete observability refers to a system where all nodes are directly observable or calculable.

The incomplete observability refers to a system where some nodes are not observable.

To assure the total observability of the network certain rules must be respected:

All nodes neighboring a node with a PMU are observable (figure2).

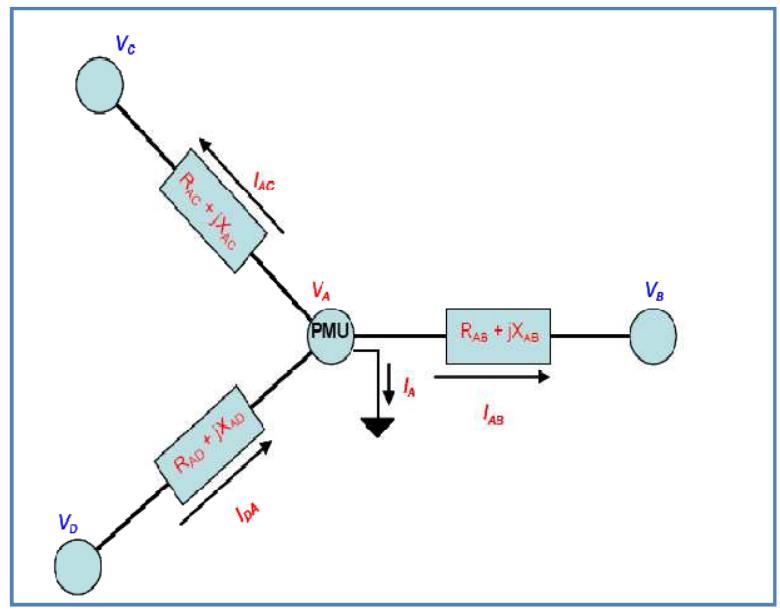

Figure 2. Example of the first rule of observability.

If a node with zero injection (node which does not inject any power into the network) is observable and all neighboring nodes are observable except one, this last one becomes observable (figure 3 ).

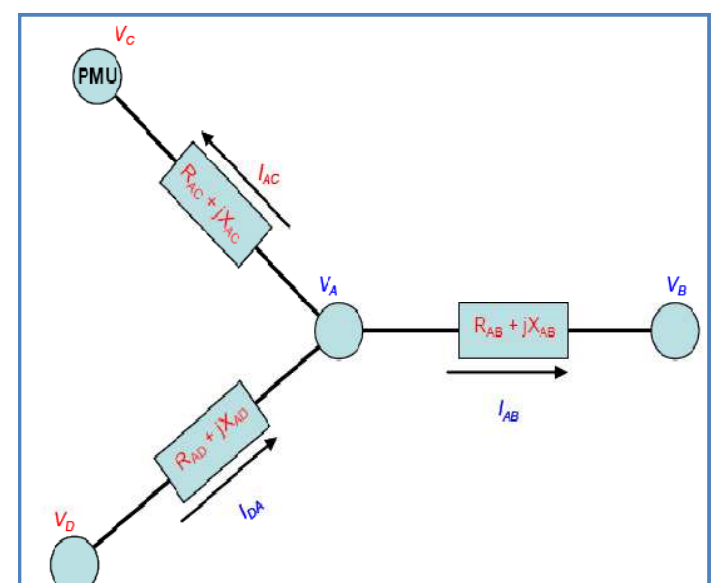

Figure 3. Example of the second rule of observability

If all neighboring nodes to a zero injection node are observable, then this node is also observable (figure4).

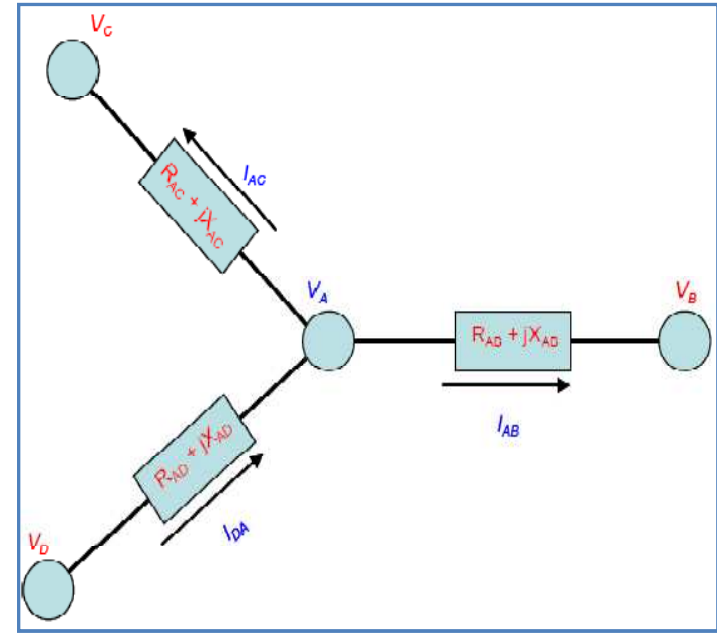

Figure 4. Example of the third rule of observability.

Given the high cost of phase measurement unit, the number of PMUs must be minimized. The goal is to determine the optimal location of PMUs ensuring total observability.

\section{Problem Formulation}

\subsection{Cost Function Formulation}

The installation minimal cost F (X) [5], is directly linked to a minimal number of Placing PMUs.

The minimization function may take the following form:

$$
\mathrm{F}(\mathrm{X})=\operatorname{Min} \sum \mathrm{Ci} * \mathrm{xi}
$$

With:

$\mathrm{C}_{\mathrm{i}}$ : The cost to install a PMU at the $\mathrm{i}^{\text {th }}$ bus.

$$
x_{i}:\left\{\begin{array}{l}
1 \text { for a node } i \text { with a PMU } \\
0 \text { otherwise }
\end{array}\right.
$$

\subsection{Observability Constraints Formulation}


The definition of observability constraints is studied in two cases as follows:

a. Case of a network with no zero injection nodes

Let the function Cost $=\mathrm{F}(\mathrm{X})$ [5] to minimize under the constraints $g(X)$ as $g(X) \geq I$

Where $g(X)=A \times X$ is a series of equations representing the system topology. The matrix A between two nodes $\mathrm{k}$ and $\mathrm{m}$ is defined as follows:

$$
A_{k, m}= \begin{cases}1 & \text { If } k=m \\ 1 & \text { If } k \text { et } m \text { are connected } \\ 0 & \text { Otherwise }\end{cases}
$$

The vector $\mathrm{I}$ is the unit vector of dimension $(\mathrm{N} \times 1)$ with $\mathrm{N}$ the total number of network node.

b. Case of a network with zero injection nodes

The study of this case is done using the matrix development method.

Solving the equation $\mathrm{A} \times \mathrm{X} \geq \mathrm{I}$ will undergo some transformations to get a faster and easier resolution by having the form :

$$
\mathrm{A} 1 \times \mathrm{X} \geq \mathrm{b} \text { then } \mathrm{A} 1 \times \mathrm{X}=\mathrm{T} \text { cont } \times \mathrm{P} \times \mathrm{A} \times \mathrm{X} \geq \mathrm{b}
$$

With:

$\mathbf{T}_{\text {cont }}$ : constraint matrix related to nodes with no zero injection, it is as follows:

$$
T_{\text {cont }}=\left[\begin{array}{cc}
I_{M * M} & 0 \\
0 & T_{i n j}
\end{array}\right]
$$

$\mathbf{T}_{\text {inj }}$ : constraint matrix related to the nodes with zero injection, of dimension $[\mathbf{x} \times \mathbf{y}]$ with $\mathbf{x}$ represents the number of the nodes with zero injection and $\mathbf{y}$ represents the number of the nodes connected to zero injection nodes +1 (without repetation of the nodes which have more than one connection).

$$
A_{1}[i, j]=\left\{\begin{array}{c}
1, \text { If } k=m, i=1, \ldots, m \\
1, \text { If } k \text { et } \text { mare connected }, j=1, \ldots, k \\
0, \text { Otherwise }
\end{array}\right.
$$

m: numbers of nodes with zero injection.

$\mathrm{k}$ : numbers of nodes connected to the zero injection nodes.

I: identity matrix $[\mathrm{M} \times \mathrm{M}]$, where $\mathrm{M}$ represents the number of nodes not connected to the zero injection nodes.

$P$ : Is a permutation matrix between old and new constraints with a dimension of $[\mathrm{N} \times \mathrm{N}]$, where $\mathrm{N}$ represents the total number of nodes in the system; ie it is the identity matrix except with disordered rows, we put the rows of the nodes with no connection to any zero injection node at the beginning, and keep the organization of the columns.

$$
P_{k, m}=\left\{\begin{array}{cl}
1 & \text { If } k=m \\
0 & \text { Otherwise }
\end{array}\right.
$$

$\mathrm{b}$ : vector of numbers of PMUs needed to ensure the observability of system, size $(a \times 1)$ with a is the number of constraints.

$$
b=\left\{\begin{array}{cc}
1 & \text { if node has no connection with the } \\
\text { zero injection node } & \text { Otherwise }
\end{array}\right.
$$

For the case of a network with a no zero injection node, the limit number of PMUs needed to assure the observability of this node is equal to 1 .

In the case of a network with a zero injection node the limit number of PMUs needed to assure the observability of this node is directly related to the number of nodes to which it is connected.

For example, for a node $\mathrm{K}$, with zero injection, related to the nodes $r, t$ and $q$, its constraint of observability is: $g \geq 3$, since it is related to three nodes $(r, t, q)$.

The formulated problem is a mono-objective optmisation problem with constraints and that could be solved using the genetic algorithms.

\section{Genetic Algorithms}

The genetic algorithms are stochastic techniques of optimization which try to imitate the processes of natural evolution of the species and the genetics. They act on a population of individuals subjected to a Darwinian selection: the individuals, known as parents, best adapted to their environment survive and can reproduce. They are then subjected to mechanisms of recombination similar to those of the genetics. Exchanges of genes between parents results in the creation of new individuals, known as children.

The genetic algorithms basically differ from the other methods in research of the optimum:

They act on a set of configurations (populations) and not a single point.

They use only the values of the function to be optimized, not its derivative or other auxiliary information.

They use probabilistic transition rules (no deterministic).

The following functional flow chart illustrates the structure of the genetic algorithm [11].

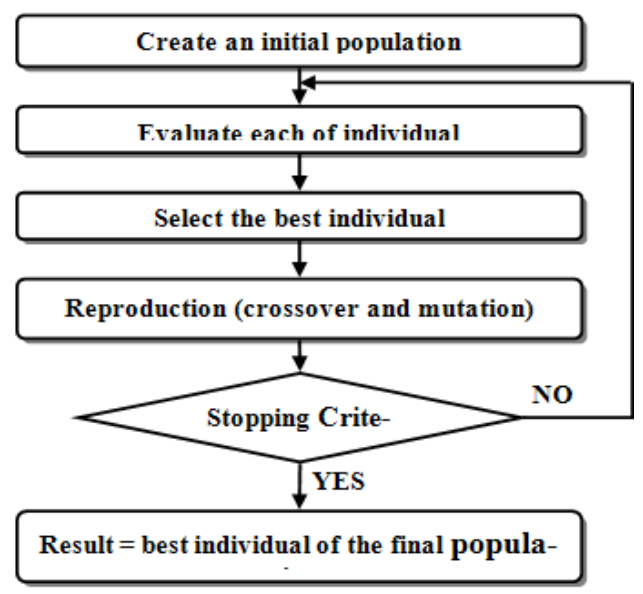

Figure 5. Structure of the genetic algorithm. 
The definitions of different steps of the genetic algorithm are defined as follows:

Selection: This operator's role is to detect which individuals of the current population will be allowed to reproduce ("parents"). Selection is based on the quality of individuals, estimated using a function called "fitness," "evaluation function," or "performance." The methods of selection are: by rank, Steady State, by casino roulette, N / 2 elitism, and tournament.

Evaluation: This step is to calculate (or estimate) the quality of newly created individuals. Here, and only here, that the function to be optimized intervenes. No assumptions are made about the function itself, except that it must be the basis for selection process.

Reproduction: The selected parents are used to generate descendants. The two principal operations are the crossover, which combine genes of 2 parents, and the mutation which consists of a light disturbance of the genome. These operations are applied arbitrarily using two parameters; the probability of crossing and the probability of mutation. These probabilities are important parameters, which considerable influence in the quality of final results.

There are several types of crossings and mutations:

Crossover : binary crossing, real crossing, arithmetic crossing.

Mutation : binary mutation, real mutation, uniform mutation, non uniform mutation.

Crossover example:

Before crossover:

\begin{tabular}{llllllllll}
\hline Parent1 & 1 & 1 & 0 & 1 & 1 & 0 & 0 & 1 & 0 \\
Parent2 & 1 & 1 & 0 & 1 & 1 & 1 & 1 & 0 & 0 \\
\hline
\end{tabular}

After crossover:

\begin{tabular}{llllllllll}
\hline Child1 & 1 & 1 & 0 & 1 & 1 & 1 & 1 & 0 & 0 \\
Child2 & 1 & 1 & 0 & 1 & 1 & 0 & 0 & 1 & 0 \\
\hline
\end{tabular}

Mutation example:

Before mutation:

\begin{tabular}{llllllllll}
\hline Parent1 & 1 & 1 & 0 & 1 & 1 & 0 & 0 & 1 & 0 \\
\hline
\end{tabular}

After mutation:

\begin{tabular}{lllllllll}
\hline Child1 1 & 1 & 0 & 0 & 1 & 0 & 0 & 1 & 1 \\
\hline
\end{tabular}

Stopping criteria: Stopping the process at the right time is essential from a practical point of view. If there is little or no information about the targeted value of the desired optimum (otherwise stopping happens when this value is achieved by the best individual of the current population), it is crucial to know when to stop the evolution.

In this article, the genetic algorithm with binary coding is employed where each placement of PMU corresponds to binary set with the length equal to the number of node in the network.

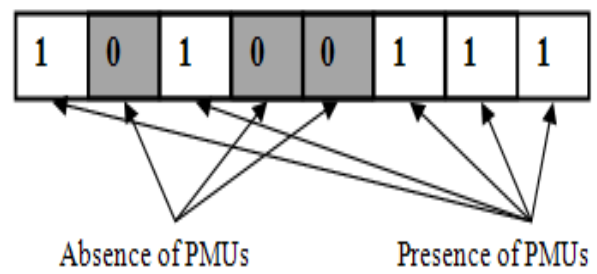

\section{Simulation and Results}

The algorithm of PMUs optimal placement is tested on three IEEE test networks: IEEE 14 bus, IEEE 30 bus and IEEE 118 bus [8].

\subsection{IEEE 14 Bus}

The structure of the network IEEE 14 bus is represented by figure 6 where the black spot close to bus 7 indicates a bus with zero injection. The data of the lines and the buses are given by table 1 .

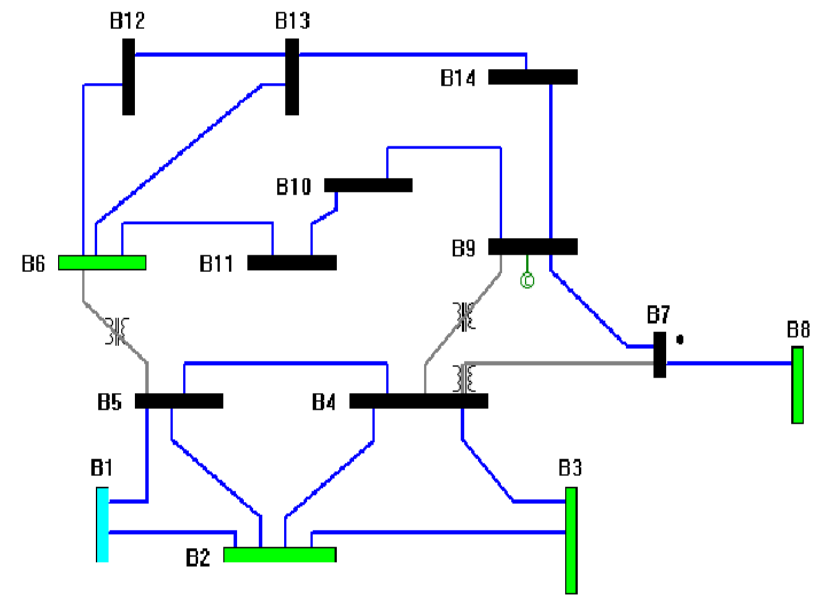

Figure 6. Structure of System 14 bus.

Table 1. Data about the IEEE System 14 bus.

\begin{tabular}{llll}
\hline System & $\begin{array}{l}\text { Number of } \\
\text { connections }\end{array}$ & $\begin{array}{l}\text { Number of buses } \\
\text { with zero injection }\end{array}$ & $\begin{array}{l}\text { Number of buses with } \\
\text { no zero injection }\end{array}$ \\
\hline 14 bus & 20 & 1 & 7 \\
\hline
\end{tabular}

\subsubsection{Case with No Zero Injection Bus}

The results of the simulation are given by the following table:

Table 2. results of network with no zero injection buses.

\begin{tabular}{lll}
\hline & no zero injection buses \\
\hline & Number of PMUs & PMUs placement \\
\hline $\begin{array}{l}\text { Reference solution } \\
{[8,9,10,11]}\end{array}$ & 4 & $2-6-7-9$ \\
$\begin{array}{l}\text { Solution of } \\
\text { genetic algorithm }\end{array}$ & 4 & $2-6-7-9$ \\
\hline
\end{tabular}




\subsubsection{Case with Zero Injection Buses}

The results of the simulation are given by the following table:

Table 3. results of network with zero injection buses.

\begin{tabular}{lll}
\hline & With zero injection buses \\
\hline & Number of PMUs & PMUs placement \\
\hline $\begin{array}{l}\text { Reference solution } \\
{[8,9,10,11]}\end{array}$ & 3 & $\mathbf{2 - 6 - 9}$ \\
$\begin{array}{l}\text { Solution of } \\
\text { genetic algorithm }\end{array}$ & 3 & $\mathbf{2 - 6 - 9}$ \\
\hline
\end{tabular}

The genetic algorithm produced the same results that are found by the other algorithms published in the IEEE articles; therefore it is functional for the system 14-bus.

\subsection{IEEE $30 \mathrm{BUS}$}

The structure of the network IEEE 30 bus is represented by figure 7 . The data of the lines and the buses are given by table 4

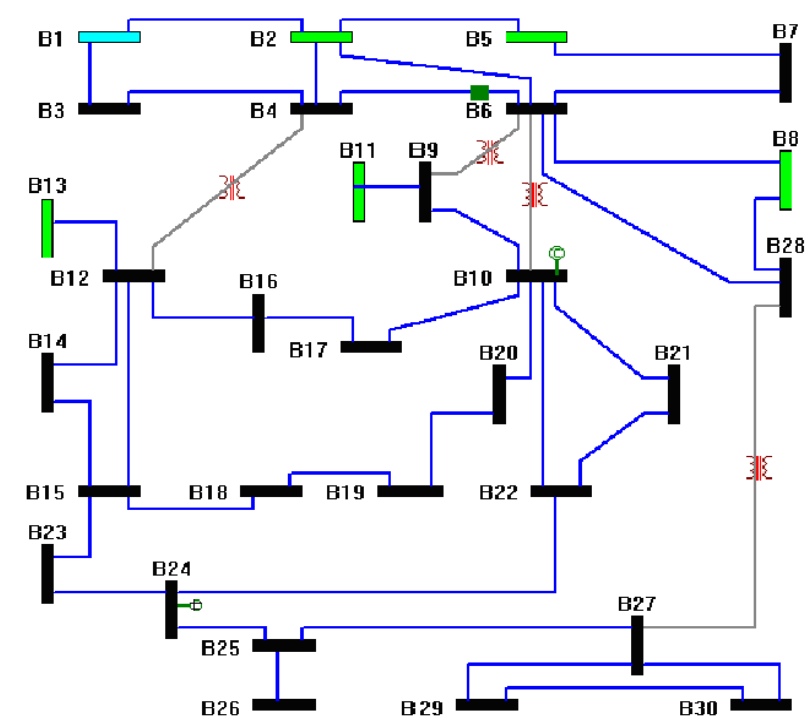

Figure 7. Structure of System 30 bus.

Table 4. Data about the IEEE System 30 bus.

\begin{tabular}{llll}
\hline System & $\begin{array}{l}\text { Number } \\
\text { of connections with zero injection }\end{array}$ & $\begin{array}{l}\text { Number of buses } \\
\text { zero injection }\end{array}$ & $\begin{array}{l}\text { Number of } \\
\text { buses with no } \\
\text { zero }\end{array}$ \\
\hline 30 bus & 41 & 5 & $6-9-11-25-28$ \\
\hline
\end{tabular}

\subsubsection{Case with No Zero Injection Buses}

The results of the simulation are given by the following table5.

Table 5. results of network with no zero injection buses.

\begin{tabular}{|c|c|c|}
\hline \multicolumn{3}{|c|}{ no zero injection buses } \\
\hline & Number of PMUs & PMUs placement \\
\hline $\begin{array}{l}\text { Reference solution } \\
{[8,9,10,11]}\end{array}$ & 10 & $\begin{array}{l}2-4-6-9-10-12-15-18- \\
25-27\end{array}$ \\
\hline $\begin{array}{l}\text { Solution of } \\
\text { genetic algorithm }\end{array}$ & 10 & $\begin{array}{l}2-4-6-9-10-12-15-19- \\
25-27\end{array}$ \\
\hline
\end{tabular}

The genetic algorithm produces the same number of PMUs as that of the references, but the localizations of PMUs are not the same.

\subsubsection{Case with Zero Injection Buses}

The results of the simulation are given by the following table:

Table 6. results of network with zero injection buses.

\begin{tabular}{lll}
\hline & \multicolumn{2}{l}{ With zero injection buses } \\
\hline & Number of PMUs & PMUs placement \\
\hline $\begin{array}{l}\text { Reference solution } \\
{[8,9,10,11]}\end{array}$ & 7 & $2-4-6-9-10-12-15-18-$ \\
Solution of & & $25-27$ \\
genetic algorithm & 7 & $2-4-6-9-10-12-15-19-$ \\
& & $25-27$ \\
\hline
\end{tabular}

\subsection{IEEE 118 BUS}

The structure of the network IEEE 118 bus is represented by figure 8 . The data of the lines and the nodes are given by table 7

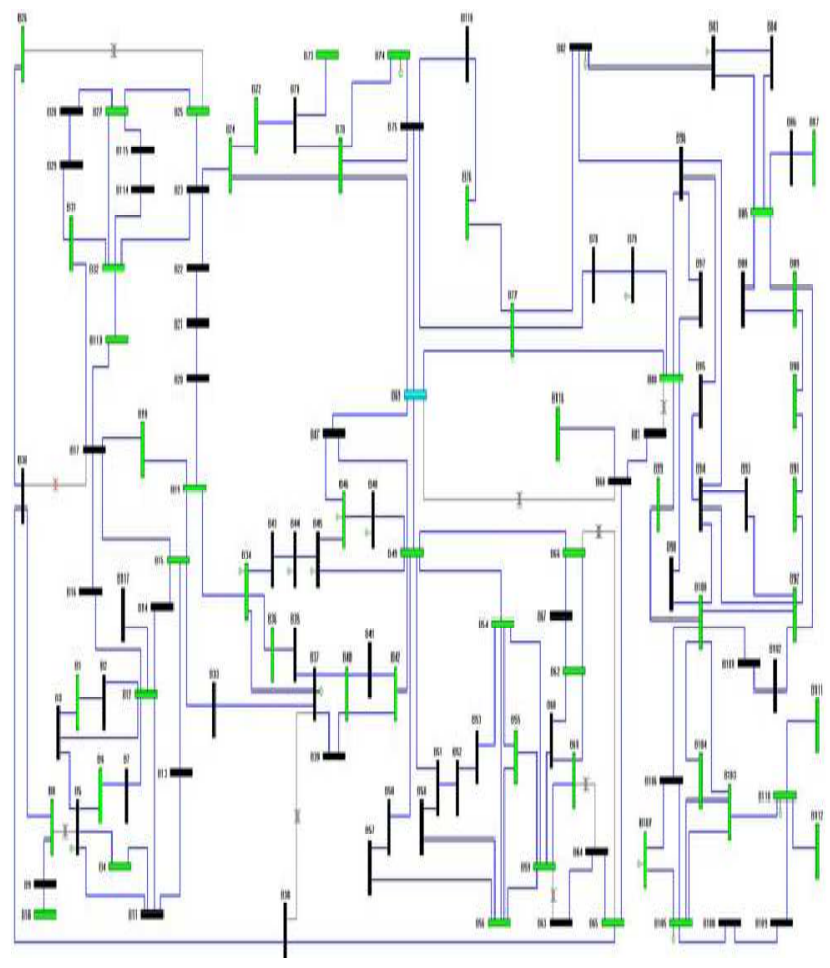

Figure 8. Structure of System 118 bus.

Table 7. Data about the IEEE System 118 bus.

\begin{tabular}{llll}
\hline System & $\begin{array}{l}\text { Number } \\
\text { of connections }\end{array}$ & $\begin{array}{l}\text { Number of buses } \\
\text { with zero injection }\end{array}$ & $\begin{array}{l}\text { Number of } \\
\text { buses with no } \\
\text { zero injection }\end{array}$ \\
\hline \multirow{2}{*}{118 nodes } & 179 & 10 & $\begin{array}{l}5-9-30-37-38-63-64- \\
68-71-81\end{array}$ \\
\hline
\end{tabular}




\subsubsection{Case with No Zero Injection Buses}

The results of the simulation are given by the following table:

Table 8. results of network with no zero injection buses 118 .

\begin{tabular}{|c|c|c|}
\hline & \multicolumn{2}{|c|}{ no zero injection buses } \\
\hline & $\begin{array}{l}\text { Number } \\
\text { of PMUs }\end{array}$ & PMUs placement \\
\hline $\begin{array}{l}\text { Reference } \\
\text { solution } \\
{[8,9,10,11]}\end{array}$ & 32 & $\begin{array}{l}2-5-9-11-12-17-21-24-25-28-34-37 \\
-40-45-49-52-56-62-63-68-73- \\
75-77-80-85-86-90-94-101-105-110-114\end{array}$ \\
\hline $\begin{array}{l}\text { Solution } 1 \\
\text { of genetic } \\
\text { algorithm }\end{array}$ & 32 & $\begin{array}{l}3-5-9-12-15-17-21-23-25-28-34- \\
37-40-45-49-53-56-62-64-69-71- \\
75-77-80-85-86-90-94-101-105-110-114\end{array}$ \\
\hline $\begin{array}{l}\text { Solution } 2 \\
\text { of genetic } \\
\text { algorithm }\end{array}$ & 32 & $\begin{array}{l}3-5-9-11-12-17-21-25-29-34-37 \\
-40-45-49-52-56-62-64-69-70-71- \\
77-80-85-86-90-94-101-105-110-114-118\end{array}$ \\
\hline $\begin{array}{l}\text { Solution } 3 \\
\text { of genetic } \\
\text { algorithm }\end{array}$ & 32 & $\begin{array}{l}3-5-9-12-15-17-21-23-29-30 \\
-34-37-42-45-49-53-56-62-64-69 \\
-71-75-77-80-85-86-90-94-101-105-110-115\end{array}$ \\
\hline
\end{tabular}

All the solutions are optimal, it is a problem of identification of the cost it is all.

\subsubsection{Case with Zero Injection Buses}

The results of the simulation are given by the following table:

Table 9. results of network with zero injection nodes 118.

\begin{tabular}{|c|c|c|}
\hline & \multicolumn{2}{|c|}{ With zero injection buses } \\
\hline & $\begin{array}{l}\text { Number } \\
\text { of PMUs }\end{array}$ & PMUs placement \\
\hline $\begin{array}{l}\text { Reference solution } \\
{[8,9,10,11]}\end{array}$ & 29 & $\begin{array}{l}2-8-11-12-15-19-21-27-31-32- \\
34-40-45-49-52-56-62-65-72-75 \\
-77-80-85-86-90-94-101-105-110\end{array}$ \\
\hline $\begin{array}{l}\text { Solution } 1 \text { of } \\
\text { genetic } \\
\text { algorithm }\end{array}$ & 29 & $\begin{array}{l}3-8-12-15-17-20-23-29-34-40-45-49- \\
52-56-62-65-70-75-77-80-85-87-89- \\
92-96-100-105-110-115\end{array}$ \\
\hline $\begin{array}{l}\text { Solution } 2 \text { of } \\
\text { genetic } \\
\text { algorithm }\end{array}$ & 29 & $\begin{array}{l}3-8-12-15-17-21-27-29-32-34 \\
-40-43-46-50-51-54-62-64-70- \\
75-77-80-85-86-91-94-101-105-110\end{array}$ \\
\hline $\begin{array}{l}\text { Solution } 3 \text { of } \\
\text { genetic } \\
\text { algorithm }\end{array}$ & 29 & $\begin{array}{l}3-8-11-12-17-21-24-27-28-32 \\
-34-38-40-45-49-52-56-59-66- \\
75-77-80-85-86-90-94-101-105-110\end{array}$ \\
\hline
\end{tabular}

\subsection{GA Effects}

Table 3, table 6 and the table 9 respectively of 14 bus system, 30 bus system and 118 bus system present the reduced number of PMUs, which allows for a minimization of the cost function with an adjustment in the depths of unobservability of buses.

\section{Conclusion}

The problem of optimal placement of PMUs (Phasor measurement Units) in an electrical power network is defined using certain constraints to guarantee a total observability of the network and to minimize the cost.

It is, thus, a problem of mono-objective optimization where the genetic algorithm is proposed as a method of study. The effectiveness and the flexibility of this algorithm are checked by results of simulation on IEEE test networks.

\section{References}

[1] B. Xu and A. Abur, "Optimal placement of phasor measurement units for state estimation," Final Project Report, PSERC, Oct. 2005.

[2] B. Xu and A. Abur, "Observability analysis and measurement placement for systems with PMUs," in proc. IEEE Power Eng. Soc. Power Systems Conf. Expo., Oct. 2004, pp. 943-946.

[3] A. Abur and A. G. Exposito, Power System State Estimation: Theory and Implementation. New York: Mercel Dekker, 2004.

[4] B. Milosevic, M. Begovic, "Nondominated sorting genetic algorithm for optimal phasor measurement placement", IEEE Transactions on. Power Systems Vol.18, No.1, Feb. 2003, pp. 69-75.

[5] Bei Gou, "Generalized Integer Linear Programming Formulation for Optimal PMU Placement," IEEE TRANSACTIONS ON POWER SYSTEMS, VOL. 23, NO. 3, AUGUST 2008.

[6] IEEE Working Group H-7, "Synchronized Sampling and Phasor Measurements for Relaying and Control", IEEE Transactions on Power Delivery, Vol. 9, No.1, January 1994, pp. 442-452.

[7] IEEE Working Group H-8, "IEEE Standard for Synchrophasors for Power Systems", IEEE Transactions on Power Delivery, Vol. 13, No. 1, January 1998.

[8] Sanjay Dambhare, "Optimal zero Injection Considetrations in PMU Placement : An ILP Approach," 16th PSCC, Glasgow, Scotland, July 14-18,2008.

[9] J.R.Altman, "A Practical Comprehensive Approach to PMU Placement for Full Observability", Master of Science In Electrical Engineering, January 28, 2007 Blacksburg, Virginia.

[10] T.A.Baldwin, L. Mili M. B. Boisen, Jr. R. Adapa, "Power system observability with minimal phasor measurement placement", IEEE Transaction. on Power Systems, Vol. 8, No. 2, May 1993, pp 2381- 2388.

[11] CHIH-WEN LIU, "Genetic Algorithms as a Reactive Power Source Dispatching Aid Genetic Algorithms as a Reactive Power Source Dispatching Aid," Proc. Natl. Sci. Counc. ROC(A). Proc. Natl. Sci. Counc. ROC(A). 